\title{
“On Water" Sonochemical Multicomponent Synthesis of Novel Bioactive 1,1'-(Aryl)bis(2-(cyclohexylamino)-2-oxoethane-1,1-diyl) Di(alkanoates and benzoates)
}

\author{
Manouchehr Mamaghani, ${ }^{1}$ Nosrat O. Mahmoodi, ${ }^{1}$ Farhad Shirini, ${ }^{1}$ \\ Abbas Azimi-Roshan, ${ }^{1}$ and Azam Monfared ${ }^{2}$ \\ ${ }^{1}$ Department of Chemistry, Faculty of Sciences, University of Guilan, P.O. Box 41335-1914, Rasht, Iran \\ ${ }^{2}$ Department of Chemistry, University of Payamnoor of Tehran, P.O. Box 1659639884, Tehran, Iran
}

Correspondence should be addressed to Manouchehr Mamaghani; mchem41@gmail.com

Received 19 May 2013; Revised 9 September 2013; Accepted 30 September 2013

Academic Editor: Marijan Kocevar

Copyright (C) 2013 Manouchehr Mamaghani et al. This is an open access article distributed under the Creative Commons Attribution License, which permits unrestricted use, distribution, and reproduction in any medium, provided the original work is properly cited.

\begin{abstract}
An expeditious, efficient, and ecofriendly ultrasound-promoted synthesis of a series of novel 1,1' -(aryl)bis(2-(cyclohexylamino)2-oxoethane-1,1-diyl) di(alkanoate and benzoate) derivatives $(\mathbf{6} \mathbf{a}-\mathbf{k})$, as useful building block candidates for natural products and drugs, is reported. The target compounds were prepared by the reaction of alkyl or aryl carboxylic acid, various bisaldehydes and isocyanocyclohexane in water using one-pot isocyanide-based multicomponent approach. This efficient protocol without using any catalyst furnished the products in short reaction times (4-5 min) and excellent yields (87-96\%). The antibacterial activity of the selected products was also examined. Some products showed promising activities.
\end{abstract}

\section{Introduction}

Organic transformations in aqueous solvents are not new to organic chemists; the earliest example dated back to 1882 when Baeyer and Drewsen reported the synthesis of indigo in aqueous acetone [1]. Water possesses many unique physical and chemical properties, and considerable rate acceleration is often observed in reactions carried out under on water conditions over those in organic solvents $[2,3]$. These specified properties are the consequence of the unique structure of water $[4,5]$. Considerable efforts have been directed at understanding the physical nature of the rate acceleration of water-based reactions. Possible source of rate increase such as hydrophobic hydration offering more favorable environment for the transition state (TS) relative to the reactants enhanced $\mathrm{H}$-bonding in the transition state compared to that in the reactants; high cohesive energy density of water and enforced hydrophobic interactions have been emphasized [6]. The use of water as a solvent for organic transformations offers several "green chemistry" benefits [7]. Other advantages of on water reactions include the safety and almost zero cost of water relative to organic solvents and the ease of product isolation. A significant example of an on water accelerated cycloaddition reaction between quadricyclane and dimethyl azodicarboxylate was described by Narayan and coworkers. This reaction took $10 \mathrm{~min}$ (on water) and 5 days (in toluene) to reach completion [8]. In addition to the rate acceleration or increase in yields, high stereo- and regioselectivity were also obtained for asymmetric aldol reactions and certain Diels-Alder reactions by performing emulsion reactions on water [6].

On the other hand, MCRs are by far the most versatile reactions in terms of scaffolds and number of accessible compounds and have gained prominence with the advent of combinatorial chemistry and related library-synthesis strategies in the last two decades [9-12]. In particular isocyanidebased multicomponent reactions (IMCRs) have been of particular interest due to their wide applications in the synthesis of natural products, lead compounds in the drug discovery 
process, and other targets of interest [13-19]. Among the isocyanide-based multicomponent reactions, the Passerini reaction is the most important one which incorporates every atom in the starting material in the product. Consequently, there is no intrinsic chemical waste associated with the reaction, therefore achieving $100 \%$ atom economy [20, 21].

Recently, the use of ultrasonic irradiation as clean, green, and environmentally benign route to activate organic reactions has attained greater value, compared to conventional heating. It has been used for a wide variety of organic reactions, such as sterically congested Passerini reactions [22], oxidations, reductions, cleavage of epoxides, multicomponent reactions, synthesis of ionic liquids, and N-heterocyclic compounds [23-25].

In this report, we have used Passerini reaction for isocyanide-based multicomponent synthesis of novel $1,1^{\prime}$-(aryl) bis(2-(cyclohexylamino)-2-oxoethane-1,1-diyl) di(alkanoate and benzoate) derivatives under ultrasonic irradiation and without catalyst, performed on water under the conditions defined by Narayan and coworkers $[3,8]$.

\section{Experimental}

2.1. General. For the ultrasound reactions, ultrasound apparatus Astra 3D $(9.5 \mathrm{~L}, 45 \mathrm{kHz}$ frequency, input power with heating, $305 \mathrm{~W}$, number of transducers, 2) from TECNOGAZ was used. Melting points were measured on an Electrothermal 9100 apparatus. FT-IR spectra were determined on a Shimadzo FT-IR spectrometer. ${ }^{1} \mathrm{H}$ NMR and ${ }^{13} \mathrm{C}$ NMR spectra were recorded on a 400 or $500 \mathrm{MHz}$ Bruker DRX400 in $\mathrm{CDCl}_{3}$ or DMSO- $\mathrm{d}_{6}$ as solvent and TMS as an internal standard. Elemental analyses were done on a CarloErba EA1110 CNOS analyzer and agreed with the calculated values. Chemicals were purchased from Merck and Aldrich. All solvents used were dried and distilled according to the standard procedures.

2.2. Synthesis of $\mathbf{6} \boldsymbol{a}-\boldsymbol{k}$. A mixture of alkyl and aryl carboxylic acid (5a-h) (1.0 mmol), cyclohexyl isocyanide (3) $(1.0 \mathrm{mmol}$, $0.109 \mathrm{~g})$, bisaldehydes $(4 \mathbf{a}-\mathbf{c})(0.5 \mathrm{mmol})$, and $\mathrm{H}_{2} \mathrm{O}(5 \mathrm{~mL})$ were placed into Pyrex glass open vessel and irradiated in a water bath under silent condition by ultrasound $(45 \mathrm{kHz})$ at room temperature for the required reaction times. Initially the reaction occurred under heterogeneous condition, and the solid product thus formed was isolated by filtration, washed with $3 \times 1 \mathrm{~mL}$ of $10 \% \mathrm{NaHCO}_{3}$ and $2 \times 1 \mathrm{~mL}$ water, and dried under high vacuum. The product was recrystallized from ethanol to produce $1,1^{\prime}$-(aryl)bis(2-(cyclohexylamino)2-oxoethane-1,1-diyl) di(alkanoate and benzoate) derivatives $(\mathbf{6 a - k})$ as pure crystalline products in $87-96 \%$ yields (Table 2).

2.2.1. 6a: White Solid. Mp 212-214 ${ }^{\circ} \mathrm{C}$; FT-IR $\left(\mathrm{KBr}, \mathrm{cm}^{-1}\right)$ $v_{\max } 3295,3070,2920,2845,1735,1655,1540,1055 ;{ }^{1} \mathrm{H}$ NMR $\left(400 \mathrm{MHz} \mathrm{CDCl}_{3}\right) \delta_{\mathrm{H}}: 1.18(6 \mathrm{H}, \mathrm{m}), 1.36(4 \mathrm{H}, \mathrm{m}), 1.69(8 \mathrm{H}$, m), $1.92(4 \mathrm{H}, \mathrm{d}$, br., $J=15.4 \mathrm{~Hz}), 2.19(4 \mathrm{H}, \mathrm{s}), 3.78(2 \mathrm{H}, \mathrm{m})$, $6.02(2 \mathrm{H}, \mathrm{d}, J=8.8 \mathrm{~Hz}, \mathrm{NH}), 6.05(2 \mathrm{H}, \mathrm{s}), 7.43(4 \mathrm{H}, \mathrm{s}) ;{ }^{13} \mathrm{C}$ NMR $\left(100 \mathrm{MHz}, \mathrm{CDCl}_{3}\right) \delta_{\mathrm{C}}: 21.1,24.8,25.4,32.9,33.0,48.3$,
75.0, 127.8, 136.5, 166.9, 169.0; Anal. Calcd. for $\mathrm{C}_{26} \mathrm{H}_{36} \mathrm{~N}_{2} \mathrm{O}_{6}$ : C, 66.08; H, 7.68; N, 5.93. Found: C, 66.16; H, 7.76; N, 6.05 .

2.2.2. 6b: White Solid. Mp $232-233^{\circ} \mathrm{C}$; FT-IR $\left(\mathrm{KBr}, \mathrm{cm}^{-1}\right)$ $v_{\max } 3300,3080,2920,2850,1745,1660,1540,1080 ;{ }^{1} \mathrm{H}$ NMR $\left(400 \mathrm{MHz} \mathrm{CDCl}_{3}\right) \delta_{\mathrm{H}}: 1.16(4 \mathrm{H}, \mathrm{m}), 1.20(6 \mathrm{H}, \mathrm{m}), 1.37(4 \mathrm{H}$, m), $1.63(4 \mathrm{H}, \mathrm{m}), 1.72$ (4H, d, br., $J=13.2 \mathrm{~Hz}), 1.91$ (4H, d, br., $J=11.6 \mathrm{~Hz}), 2.47(4 \mathrm{H}, \mathrm{m}), 3.79(2 \mathrm{H}, \mathrm{m}), 6.02(2 \mathrm{H}, \mathrm{d}, J=$ $6.4 \mathrm{~Hz}, \mathrm{NH}), 6.07(2 \mathrm{H}, \mathrm{s}), 7.43(4 \mathrm{H}, \mathrm{s}) ;{ }^{13} \mathrm{C}$ NMR $(100 \mathrm{MHz}$, $\left.\mathrm{CDCl}_{3}\right) \delta_{\mathrm{C}}: 9.0,24.7,25.4,27.6,32.9,33.0,48.2,74.8$, 127.7, 136.5, 167.1, 172.4; Anal. Calcd. for $\mathrm{C}_{28} \mathrm{H}_{40} \mathrm{~N}_{2} \mathrm{O}_{6}$ : C, 67.05; $\mathrm{H}$, 8.05; N, 5.60. Found: C, 67.14; H, 7.98; N, 5.71.

2.2.3. 6c: White Solid. Mp $212-214^{\circ} \mathrm{C}$; FT-IR $\left(\mathrm{KBr}, \mathrm{cm}^{-1}\right)$ $v_{\max } 3300,3080,2925,2845,1745,1660,1545,1140 ;{ }^{1} \mathrm{H}$ NMR $\left(400 \mathrm{MHz} \mathrm{CDCl}_{3}\right) \delta_{\mathrm{H}}: 1.00(6 \mathrm{H}, \mathrm{m}), 1.17(6 \mathrm{H}, \mathrm{m}), 1.38(4 \mathrm{H}$, $\mathrm{m}), 1.62(2 \mathrm{H}, \mathrm{s}), 1.72(8 \mathrm{H}, \mathrm{m}), 1.92(4 \mathrm{H}, \mathrm{d}$, br., $J=10.8 \mathrm{~Hz})$, $2.43(4 \mathrm{H}, \mathrm{m}), 3.80(2 \mathrm{H}, \mathrm{m}), 6.01(2 \mathrm{H}, \mathrm{d}, \mathrm{br} ., J=6.4 \mathrm{~Hz}, \mathrm{NH})$, $6.08(2 \mathrm{H}, \mathrm{s}), 7.43(4 \mathrm{H}, \mathrm{s}) ;{ }^{13} \mathrm{C} \mathrm{NMR}\left(100 \mathrm{MHz} \mathrm{CDCl}_{3}\right) \delta_{\mathrm{C}}$ : 13.6, 18.4, 24.7, 25.4, 32.9, 33.0, 36.1, 48.2, 74.7, 127.7, 136.5, 167.1, 171.6; Anal. Calcd. for $\mathrm{C}_{30} \mathrm{H}_{44} \mathrm{~N}_{2} \mathrm{O}_{6}$ : C, 68.15; H, 8.39; N, 5.30. Found: C, 68.10; H, 8.48; N, 5.37.

2.2.4. 6d: White Solid. Mp $214-215^{\circ} \mathrm{C}$; FT-IR $\left(\mathrm{KBr}, \mathrm{cm}^{-1}\right)$ $v_{\max } 3290,3080,2940,2845,1730,1660,1540,1160 ;{ }^{1} \mathrm{H}$ NMR $\left(500 \mathrm{MHz}, \mathrm{DMSO}-\mathrm{d}_{6}\right) \delta_{\mathrm{H}}: 1.11(6 \mathrm{H}, \mathrm{m}), 1.22(6 \mathrm{H}, \mathrm{m}), 1.29$ $(6 \mathrm{H}, \mathrm{m}), 1.54(8 \mathrm{H}, \mathrm{s}), 1.59(4 \mathrm{H}, \mathrm{m}), 1.70(4 \mathrm{H}, \mathrm{m}), 2.40(4 \mathrm{H}$, $\mathrm{m}), 3.46(2 \mathrm{H}, \mathrm{s}), 5.81(2 \mathrm{H}, \mathrm{s}), 7.44(4 \mathrm{H}, \mathrm{s}), 8.10(2 \mathrm{H}, \mathrm{d}, J=$ $8.0 \mathrm{~Hz}, \mathrm{NH}) ;{ }^{13} \mathrm{C}$ NMR $\left(100 \mathrm{MHz}, \mathrm{DMSO}-\mathrm{d}_{6}\right) \delta_{\mathrm{C}}: 14.1,22.0$, 24.9, 24.94, 25.6, 26.9, 32.5, 32.6, 33.5, 48.1, 74.9, 127.6, 136.8, 167.2, 172.6; Anal. Calcd. for $\mathrm{C}_{32} \mathrm{H}_{48} \mathrm{~N}_{2} \mathrm{O}_{6}$ : C, 69.03; H, 8.68; N, 5.03. Found: C, 68.83; H, 8.59; N, 5.22.

2.2.5. 6e: White Solid. Mp $179-180^{\circ} \mathrm{C}$; FT-IR $\left(\mathrm{KBr}, \mathrm{cm}^{-1}\right)$ $v_{\max } 3270,3100,2920,2840,1730,1655,1560,1110 ;{ }^{1} \mathrm{H}$ NMR $\left(400 \mathrm{MHz}, \mathrm{DMSO}-\mathrm{d}_{6}\right) \delta_{\mathrm{H}}: 1.15(8 \mathrm{H}, \mathrm{m}), 1.54(8 \mathrm{H}, \mathrm{m}), 1.68$ $(2 \mathrm{H}, \mathrm{s}), 1.77(2 \mathrm{H}, \mathrm{s}), 3.49(2 \mathrm{H}, \mathrm{s}), 6.08(2 \mathrm{H}, \mathrm{s}), 7.57(6 \mathrm{H}$, $\mathrm{m}), 7.64(4 \mathrm{H}, \mathrm{s}), 7.71(2 \mathrm{H}$, quint., $J=6.8 \mathrm{~Hz}), 7.83(2 \mathrm{H}, \mathrm{d}$, $J=8.0 \mathrm{~Hz}, \mathrm{NH}), 8.06(4 \mathrm{H}, \mathrm{t}, J=8.0,7.2 \mathrm{~Hz}) ;{ }^{13} \mathrm{C} \mathrm{NMR}$ $\left(100 \mathrm{MHz}, \mathrm{DMSO}_{-} \mathrm{d}_{6}\right) \delta_{\mathrm{C}}: 24.8,24.9,25.6,32.5,32.7,48.2,75.7$, 127.7, 127.74, 128.0, 129.3, 129.6, 129.9, 130.1, 134.1, 136.8, 165.4, 167.1; Anal. Calcd. for $\mathrm{C}_{36} \mathrm{H}_{40} \mathrm{~N}_{2} \mathrm{O}_{6}$ : C, 72.46; $\mathrm{H}, 6.76 ; \mathrm{N}, 4.69$. Found: C, 72.58; H, 6.69; N, 4.75 .

2.2.6. 6f: White Solid. Mp 203-205 $\mathrm{C}$; FT-IR $\left(\mathrm{KBr}, \mathrm{cm}^{-1}\right)$ $v_{\max } 3295,3080,2925,2845,1730,1665,1545,1075 ;{ }^{1} \mathrm{H}$ NMR $\left(400 \mathrm{MHz}, \mathrm{DMSO}-\mathrm{d}_{6}\right) \delta_{\mathrm{H}}: 1.10(4 \mathrm{H}, \mathrm{m}), 1.20(6 \mathrm{H}, \mathrm{m}), 1.54$ $(6 \mathrm{H}, \mathrm{m}), 1.72(4 \mathrm{H}, \mathrm{m}), 2.53(6 \mathrm{H}, \mathrm{s}), 3.50(2 \mathrm{H}, \mathrm{s}), 6.06(2 \mathrm{H}$, s), $7.32(2 \mathrm{H}, \mathrm{d}, J=6.8 \mathrm{~Hz}), 7.34(2 \mathrm{H}, \mathrm{d}, J=5.6 \mathrm{~Hz}, \mathrm{NH}), 7.50$ ( $2 \mathrm{H}$, quint., $J=1.2,7.6 \mathrm{~Hz}), 7.61(4 \mathrm{H}, \mathrm{s}), 7.94(2 \mathrm{H}, \mathrm{t}, J=3.6$, $8.0 \mathrm{~Hz}), 8.26(2 \mathrm{H}, \mathrm{d}, J=7.2 \mathrm{~Hz}) ;{ }^{13} \mathrm{C}$ NMR $(100 \mathrm{MHz}$, DMSO$\left.\mathrm{d}_{6}\right) \delta_{\mathrm{C}}: 21.5,24.8,24.9,25.6,32.5,32.6,48.2$, 75.8, 126.5, 127.8, 129.4, 130.9, 132.1, 132.9, 136.7, 139.8, 166.5, 167.2; Anal. Calcd. for $\mathrm{C}_{38} \mathrm{H}_{44} \mathrm{~N}_{2} \mathrm{O}_{6}$ : C, 73.05; H, 7.10; N, 4.48. Found: C, 73.17; $\mathrm{H}, 7.16 ; \mathrm{N}, 4.45$.

2.2.7. 6g: White Solid. Mp 171- $172^{\circ} \mathrm{C}$; FT-IR $\left(\mathrm{KBr}, \mathrm{cm}^{-1}\right)$ $v_{\max } 3300,3055,2945,2865,1745,1660,1555,1110 ;{ }^{1} \mathrm{H}$ NMR 
$\left(400 \mathrm{MHz}, \mathrm{DMSO}-\mathrm{d}_{6}\right) \delta_{\mathrm{H}}: 1.20(10 \mathrm{H}, \mathrm{m}), 1.55(6 \mathrm{H}, \mathrm{m}), 1.72$ $(4 \mathrm{H}, \mathrm{m}), 3.52(2 \mathrm{H}, \mathrm{s}), 6.07(2 \mathrm{H}, \mathrm{s}), 7.50(2 \mathrm{H}, \mathrm{m}), 7.62(8 \mathrm{H}, \mathrm{m})$, $7.96(2 \mathrm{H}, \mathrm{dt}, J=7.2,0.8 \mathrm{~Hz}), 8.28(2 \mathrm{H}, \mathrm{d}, J=7.6 \mathrm{~Hz}) ;{ }^{13} \mathrm{C} \mathrm{NMR}$ $\left(100 \mathrm{MHz}, \mathrm{DMSO}-\mathrm{d}_{6}\right) \delta_{\mathrm{C}}: 24.8,24.9,25.6,32.5,32.6,48.2$, 76.3, 127.8, 127.9, 127.92, 129.5, 131.4, 132.2, 132.8, 134.1, 136.4, 164.4, 166.8; MS (EI, $70 \mathrm{eV}): \mathrm{m} / z(\%)=666(0.58)\left[\mathrm{M}^{+\bullet}+2\right]$, $664(0.9)\left[\mathrm{M}^{+\bullet}\right], 274(34), 156$ (19), 141 (36), 140 (14), 139 (100) [ $\left.\mathrm{C}_{7} \mathrm{H}_{4} \mathrm{ClO}\right], 113$ (11), $111(26)\left[\mathrm{C}_{6} \mathrm{H}_{4} \mathrm{Cl}\right], 97$ (13), 83 (18). Anal. Calcd. for $\mathrm{C}_{36} \mathrm{H}_{38} \mathrm{Cl}_{2} \mathrm{~N}_{2} \mathrm{O}_{6}$ : C, 64.96; $\mathrm{H}, 5.75 ; \mathrm{N}, 4.21$. Found: C, 64.85; H, 5.81; N, 4.26.

2.2.8. 6h: White Solid. Mp 222-224 $4^{\circ}$; FT-IR $\left(\mathrm{KBr}, \mathrm{cm}^{-1}\right)$ $v_{\max } 3257,3079,2929,2855,1723,1670,1564,1109 ;{ }^{1} \mathrm{H}$ NMR $\left(500 \mathrm{MHz}, \mathrm{CDCl}_{3}\right) \delta_{\mathrm{H}}: 1.14(6 \mathrm{H}, \mathrm{m}), 1.33(4 \mathrm{H}, \mathrm{m}), 1.62(6 \mathrm{H}$, m), $1.90(4 \mathrm{H}, \mathrm{m}), 3.79(2 \mathrm{H}$, quint., $J=3.65 \mathrm{~Hz}), 5.98(2 \mathrm{H}, \mathrm{t}$, $J=8.28 \mathrm{~Hz}), 6.31(2 \mathrm{H}, \mathrm{d}, J=8.32 \mathrm{~Hz}, \mathrm{NH}), 7.46(5 \mathrm{H}, \mathrm{m}), 7.55$ $(2 \mathrm{H}, \mathrm{m}), 7.61(2 \mathrm{H}, \mathrm{m}), 7.69(1 \mathrm{H}, \mathrm{s}), 8.08(4 \mathrm{H}, \mathrm{d}, J=7.54 \mathrm{~Hz})$; ${ }^{13} \mathrm{C} \mathrm{NMR}\left(100 \mathrm{MHz}, \mathrm{DMSO}-\mathrm{d}_{6}\right) \delta_{\mathrm{C}}: 24.8,24.9,25.0,25.7,32.4$, 32.5, 32.7, 48.1, 48.2, 75.7, 75.8, 125.6, 126.1, 127.6, 128.1, 129.0, $129.1,129.2,129.3,129.7,129.9,130.0,134.1,136.8,136.9,165.3$, 165.4, 167.0, 167.1; Anal. Calcd. for $\mathrm{C}_{36} \mathrm{H}_{40} \mathrm{~N}_{2} \mathrm{O}_{6}$ : C, 72.46; $\mathrm{H}$, $6.76 ; \mathrm{N}, 4.69$. Found: C, 72.60; H, 6.82; N, 4.66.

2.2.9. 6i: Light Yellow Solid. Mp $154-155^{\circ} \mathrm{C}$; FT-IR $\left(\mathrm{KBr}, \mathrm{cm}^{-1}\right)$ $v_{\max } 3274,3087,2929,2856,1734,1661,1538,1483,1347,1136$; ${ }^{1} \mathrm{H}$ NMR $\left(500 \mathrm{MHz}, \mathrm{CDCl}_{3}\right) \delta_{\mathrm{H}}: 0.96(4 \mathrm{H}, \mathrm{m}), 1.11(6 \mathrm{H}, \mathrm{m})$, $1.46(4 \mathrm{H}, \mathrm{m}), 1.58(4 \mathrm{H}, \mathrm{m}), 1.78(2 \mathrm{H}, \mathrm{d}$, br., $J=11.38 \mathrm{~Hz}), 3.57$ $(2 \mathrm{H}, \mathrm{s}), 6.15(2 \mathrm{H}, \mathrm{s}), 6.95(2 \mathrm{H}, \mathrm{s}, \mathrm{br} ., \mathrm{NH}), 7.33(1 \mathrm{H}, \mathrm{t}, J=$ $9.39 \mathrm{~Hz}), 7.50(2 \mathrm{H}, \mathrm{d}, J=8.0 \mathrm{~Hz}), 7.55(2 \mathrm{H}, \mathrm{t}, J=8.16 \mathrm{~Hz})$, $7.72(1 \mathrm{H}, \mathrm{s}), 8.31(4 \mathrm{H}, \mathrm{t}, J=9.26 \mathrm{~Hz}), 8.75(2 \mathrm{H}, \mathrm{s}) ;{ }^{13} \mathrm{C} \mathrm{NMR}$ $\left(100 \mathrm{MHz}, \mathrm{DMSO}-\mathrm{d}_{6}\right) \delta_{\mathrm{C}}: 24.8,24.9,25.5,32.4,32.7,48.2$, 76.4, 124.3, 126.0, 128.2, 128.6, 129.2, 131.2, 131.3, 136.0, 136.5, 148.4, 163.8, 166.7; Anal. Calcd. for $\mathrm{C}_{36} \mathrm{H}_{38} \mathrm{~N}_{4} \mathrm{O}_{10}$ : C, 62.96; H, 5.58; N, 8.16. Found: C, 62.81; H, 5.64; N, 8.23.

2.2.10. 6j: White Solid. Mp $185-187^{\circ} \mathrm{C}$; FT-IR $\left(\mathrm{KBr}, \mathrm{cm}^{-1}\right)$ $v_{\max } 3248,3076,2925,2855,1721,1658,1559,1057 ;{ }^{1} \mathrm{H}$ NMR $\left(400 \mathrm{MHz}, \mathrm{DMSO}-\mathrm{d}_{6}\right) \delta_{\mathrm{H}}: 1.09(4 \mathrm{H}, \mathrm{m}), 1.20(6 \mathrm{H}, \mathrm{m}), 1.53$ $(6 \mathrm{H}, \mathrm{m}), 1.72(4 \mathrm{H}, \mathrm{m}), 2.52(6 \mathrm{H}, \mathrm{s}), 3.50(2 \mathrm{H}, \mathrm{s}), 6.08(2 \mathrm{H}, \mathrm{d}$, $J=3.6 \mathrm{~Hz}), 7.30(2 \mathrm{H}, \mathrm{d}, J=7.6 \mathrm{~Hz}, \mathrm{NH}), 7.33(2 \mathrm{H}, \mathrm{d}, J=$ $7.2 \mathrm{~Hz}), 7.46(1 \mathrm{H}, \mathrm{m}), 7.51(2 \mathrm{H}, \mathrm{m}), 7.57(2 \mathrm{H}, \mathrm{d}, J=8.4 \mathrm{~Hz}), 7.78$ $(1 \mathrm{H}, \mathrm{s}), 7.78(1 \mathrm{H}, \mathrm{s}), 7.94(2 \mathrm{H}, \mathrm{dd}, J=7.6,1.2 \mathrm{~Hz}), 8.27(1 \mathrm{H}, \mathrm{d}$, $J=8.0), 8.29(1 \mathrm{H}, J=8.0 \mathrm{~Hz}) ;{ }^{13} \mathrm{C}$ NMR $(100 \mathrm{MHz}$, DMSO$\left.\mathrm{d}_{6}\right) \delta_{\mathrm{C}}: 21.5,21.54,24.8,24.88,24.9,25.6,32.5,32.7,48.1,48.2$, $75.8,126.0,126.3,126.4,128.0,128.2,128.94,129.0,129.5,130.8$, 130.9, 132.1, 132.9, 136.8, 139.8, 139.9, 166.5, 166.6, 167.0, 167.1; Anal. Calcd. for $\mathrm{C}_{38} \mathrm{H}_{44} \mathrm{~N}_{2} \mathrm{O}_{6}$ : C, 73.05; $\mathrm{H}, 7.10 ; \mathrm{N}, 4.48$. Found: C, 73.16; H, 7.02; N, 4.43.

2.2.11. 6k: White Solid. Mp $183-185^{\circ} \mathrm{C}$; FT-IR $\left(\mathrm{KBr}, \mathrm{cm}^{-1}\right)$ $v_{\max } 3416,3067,2933,2861,1717,1678,1595,1100 ;{ }^{1} \mathrm{H}$ NMR $\left(500 \mathrm{MHz}, \mathrm{CDCl}_{3}\right) \delta_{\mathrm{H}}: 1.14(6 \mathrm{H}, \mathrm{m}), 1.32(4 \mathrm{H}, \mathrm{m}), 1.56(6 \mathrm{H}$, $\mathrm{m}), 1.65(2 \mathrm{H}, \mathrm{m}), 1.66(2 \mathrm{H}, \mathrm{m}), 1.87(2 \mathrm{H}, \mathrm{m}), 2.03(2 \mathrm{H}, \mathrm{m}), 3.79$ $(2 \mathrm{H}, \mathrm{m}), 4.08(2 \mathrm{H}, \mathrm{m}), 4.15(2 \mathrm{H}, \mathrm{m}), 6.15(2 \mathrm{H}, \mathrm{d}, J=8.14, \mathrm{NH})$, $6.66(2 \mathrm{H}, \mathrm{s}), 6.91(2 \mathrm{H}, \mathrm{m}), 7.00(2 \mathrm{H}, \mathrm{m}), 7.33(2 \mathrm{H}, \mathrm{m}), 7.43(4 \mathrm{H}$, m), $7.55(4 \mathrm{H}, \mathrm{m}), 8.08(4 \mathrm{H}, \mathrm{m}) ;{ }^{13} \mathrm{C}$ NMR $(100 \mathrm{MHz}, \mathrm{DMSO}-$ $\left.\mathrm{d}_{6}\right) \delta_{\mathrm{C}}: 25.0,25.1,25.5,25.6,32.6,48.3,67.6,71.0,112.5,120.8$, $124.5,124.6,128.8,129.2,129.8,129.9,130.7,133.9,157.1,165.5$,
TABLE 1: Effect of various solvents in the synthesis of $\mathbf{6 a}$ under ultrasonic irradiation.

\begin{tabular}{lcc}
\hline Solvent & Time $(\min )$ & Yield $(\%)^{\mathrm{a}}$ \\
\hline $\mathrm{DMF}$ & 80 & 30 \\
$\mathrm{CH}_{2} \mathrm{Cl}_{2}$ & 85 & 25 \\
$1,4-$ Dioxane & 95 & 25 \\
$\mathrm{H}_{2} \mathrm{O}$ & $4(70)^{\mathrm{b}}$ & $90(65)^{\mathrm{b}}$ \\
\hline
\end{tabular}

${ }^{\mathrm{a}}$ Isolated yields.

${ }^{\mathrm{b}}$ The reaction at room temperature under conventional condition.

167.0; Anal. Calcd. for $\mathrm{C}_{46} \mathrm{H}_{52} \mathrm{~N}_{2} \mathrm{O}_{8}$ : C, 72.61; H, 6.89; N, 3.68. Found: C, 72.75; H, 6.97; N, 3.62.

2.3. Determination of Antimicrobial Activity. A sterilized glass tube ( $5 \mathrm{~mm}$ diameter) was used aseptically to make wells on plates. The antibacterial activity of compounds was assayed biologically using the agar well-diffusion method. A colony of each standard test organism was subcultured in order to obtain fresh bacteria on the nutrient agar plates at $37^{\circ} \mathrm{C}$ for $18 \mathrm{~h}$. For preparation of suspensions of microorganisms (0.5 McFarland), one to two colonies from each plate were dissolved in isotonic saline solution. Then MuellerHinton agar (Merck) plates were prepared according to manufacturers' instructions in order to evaluate the antibacterial activities of compounds. The sterile Mueller-Hinton agar plates were inoculated with the bacteria. 0.01 gram of test samples was dissolved in $1 \mathrm{~mL}$ dimethyl sulfoxide (DMSO) to obtain a stock solution. A concentration of $1 \mathrm{mg} / \mathrm{mL}$ or $100 \mu \mathrm{g} / 0.1 \mathrm{~mL}$ of each sample was prepared. $0.1 \mathrm{~mL}$ of prepared samples was dropped into each respective labeled well aseptically. The inoculated plates were left on the table for $1 \mathrm{~h}$ to allow each sample to diffuse into the agar. For comparison, gentamycin was used as a positive control and DMSO as a negative control. Test organism growth may be affected by the inhibitory action of the test compound, and so a clear zone around the disc appeared as an indication of the inhibition of the test organism growth. The results of our tests were presented as the inhibition zones, given in millimeters $(\mathrm{mm})$. Measurements were obtained after $24 \mathrm{~h}$ for bacteria.

\section{Results and Discussion}

In recent years due to emergence of green chemistry, removing organic solvents has become an important factor towards developing benign chemical technologies due to their high toxicity [26]. As a result, organic reactions in aqueous media have gained high priority in view of green methodology and have been regularly reviewed in the literature [3, 27-32].

We verified the effect of various solvents on the efficiency of the reaction by using synthesis of $\mathbf{6} \mathbf{a}$ as model reaction. The reaction proceeded best under aqueous condition as shown in Table 1, and none of the conventional organic solvents such as $\mathrm{CH}_{3} \mathrm{CN}, 1,4$-dioxane, $\mathrm{CH}_{2} \mathrm{Cl}_{2}$, and DMF proved satisfactory, and this may be due to unique properties of water.

In continuation of our efforts for developing benign synthetic methodologies for the production of various biologically important products [33-36], we wish to report 
TABLE 2: Synthesis of $1,1^{\prime}$-(aryl)bis(2-(cyclohexylamino)-2-oxoethane-1,1-diyl) di(alkanoates and benzoates) under ultrasound irradiation and antimicrobial activity of the products (zone of inhibition in $\mathrm{mm}$ ).

\begin{tabular}{|c|c|c|c|c|c|c|c|}
\hline Entry & Products & $\mathrm{MP}\left({ }^{\circ} \mathrm{C}\right)$ & Time (min) & Yield $(\%)^{\mathrm{a}, \mathrm{b}}$ & B. subtilis & P. aeruginosa & A. bacter \\
\hline a & & $212-214$ & 4 & 90 & $9(11)^{\mathrm{c}}(10)^{\mathrm{d}}$ & $14(14)^{\mathrm{c}}(15)^{\mathrm{d}}$ & $5(7)^{\mathrm{c}}$ \\
\hline b & & $232-233$ & 4 & 95 & $8(8)^{\mathrm{c}}(9)^{\mathrm{d}}$ & $12(13)^{\mathrm{c}}(12)^{\mathrm{d}}$ & 3 \\
\hline c & & $212-214$ & 5 & 90 & 4 & $12(11)^{\mathrm{c}}$ & 4 \\
\hline d & & $214-215$ & 5 & 87 & $6(7)^{c}(6)^{d}$ & $6(8)^{c}$ & - \\
\hline e & & $179-180$ & 4 & 96 & 4 & 4 & - \\
\hline f & & $203-205$ & 5 & 90 & $14(15)^{c}(16)^{d}$ & 4 & - \\
\hline
\end{tabular}


TABle 2: Continued.

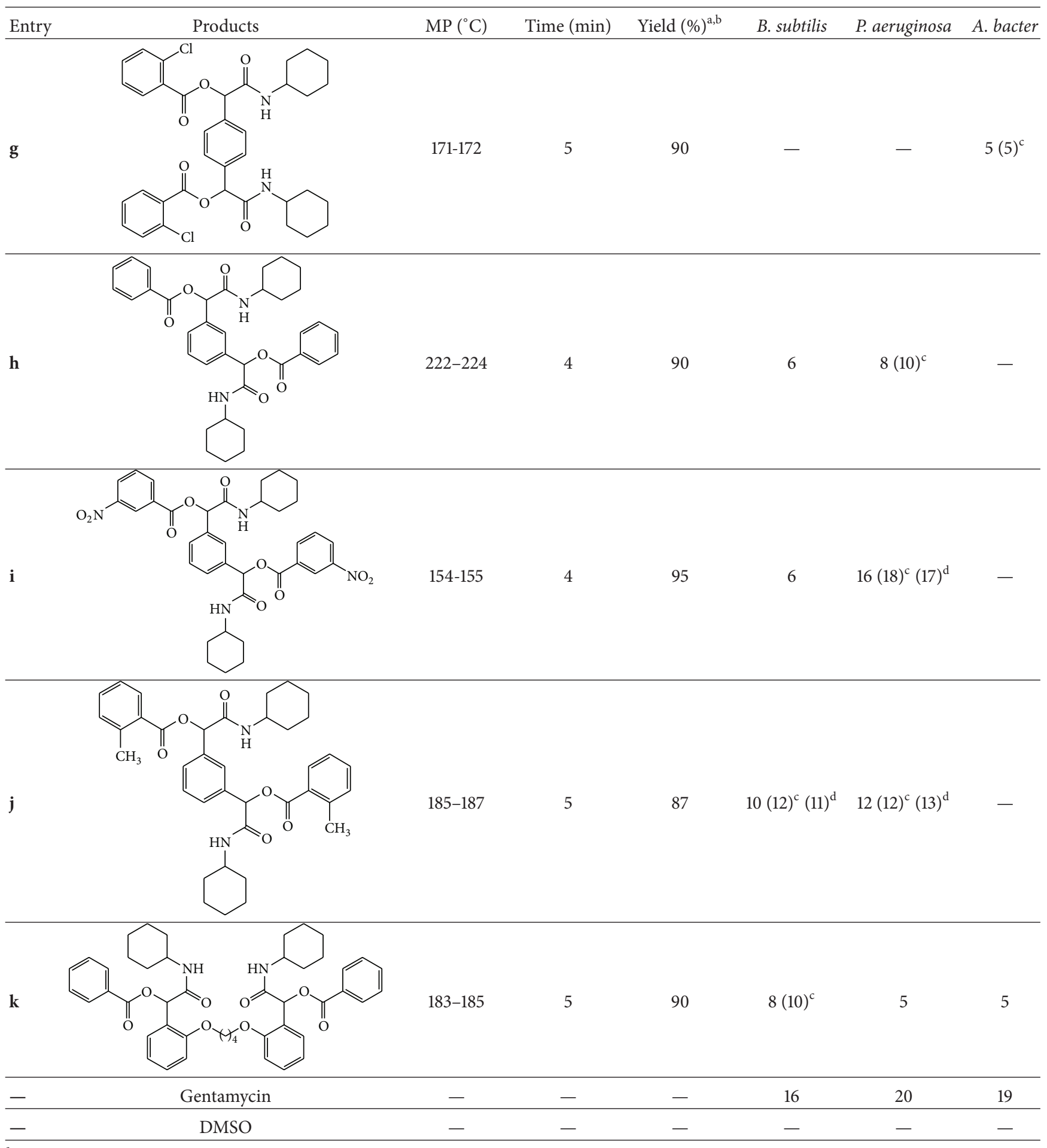

${ }^{\mathrm{a}}$ Isolated yields.

${ }^{\mathrm{b}}$ Identified by spectroscopic (FT-IR, ${ }^{1} \mathrm{H}$ NMR, and ${ }^{13} \mathrm{C}$ NMR) and elemental analyses.

${ }^{c}$ Data of duplicated experiments.

${ }^{\mathrm{d}}$ Data of triplicated experiments. 
Carboxylic acids:<smiles>CC(=O)O</smiles>

$5 a$<smiles>CCC(=O)O</smiles>

$5 b$<smiles>CCCC(=O)O</smiles>

$5 c$<smiles>CCCCC(=O)O</smiles>

5d<smiles>O=C(O)c1ccccc1</smiles>

$5 e$<smiles>Cc1ccccc1C(=O)O</smiles>

$5 f$<smiles>O=C(O)c1ccccc1Cl</smiles>

$5 \mathrm{~g}$<smiles>O=C(O)c1cccc([N+](=O)[O-])c1</smiles>

5 h

Bisaldehydes:

Isocyanide:<smiles>O=Cc1ccc(C=O)cc1</smiles><smiles>O=Cc1cccc(C=O)c1</smiles><smiles>O=Cc1ccccc1OCCOc1ccccc1C=O</smiles>

$4 \mathrm{c}$<smiles>N#CC1CCCCC1</smiles>

$4 \mathrm{a}$

4b

FIGURE 1: Building blocks for multicomponent reactions.<smiles>N#CC1CCCCC1</smiles>

3

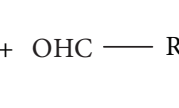

$4 a-c$

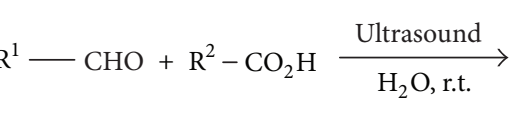

5a-h<smiles>[R]C(=O)OC([Z1]C(OC([R])=O)C(=O)NC1CCCCC1)C(=O)NC1CCCCC1</smiles>

6a-k

Scheme 1: Synthesis of 1,1'-(aryl)bis(2-(cyclohexylamino)-2-oxoethane-1,1-diyl) di(alkanoate and benzoate) derivatives.

a versatile and efficient isocyanide-based multicomponent one-pot synthesis of $1,1^{\prime}$-(aryl)bis(2-(cyclohexylamino)-2oxoethane-1,1-diyl) di(alkanoate and benzoate) derivatives $(\mathbf{6 a}-\mathbf{k})$ utilizing the reaction of alkyl and aryl carboxylic acids $(5 \mathbf{a}-\mathbf{h})(1 \mathrm{mmol})$, isocyanocyclohexane $(\mathbf{3})(1 \mathrm{mmol})$, and bisaldehydes $(\mathbf{4 a}-\mathbf{c})(0.5 \mathrm{mmol})$ (Figure 1$)$ in water under ultrasonic irradiation (Scheme 1). This rapid method produced the products in short reaction times $(4-5 \mathrm{~min})$ and excellent yields (87-96\%) (Table 2). The structures of the products were deduced from their elemental analyses and spectroscopic data.

In this study, because of the presence of the chiral carbon in the molecules, despite the symmetry of the molecules, in the ${ }^{13} \mathrm{C}$ NMR spectrum, a number of cyclohexyl carbons appeared as two distinct peaks at the region of $24-33 \mathrm{ppm}$. It is worth noting that ${ }^{1} \mathrm{H}$ NMR and FT-IR spectra as well as elemental analysis all confirmed the structure of the $1,1^{\prime}$-(aryl)bis(2-(cyclohexylamino)-2-oxoethane-1,1-diyl) di(alkanoate and benzoate) derivatives $(\mathbf{6 a}-\mathbf{k})$.

A plausible mechanism for the formation of the products $(\mathbf{6 a}-\mathbf{k})$ can be visualized in Scheme 2.

The in vitro antibacterial activities of compounds $6 \mathbf{a}-\mathbf{k}$ were evaluated against gram-positive and gram-negative bacteria using the cultures of three different standard microorganisms: Acinetobacter calcoaceticus ATCC 23055 and Pseudomonas aeruginosa ATCC 9027 as gram-negative models 


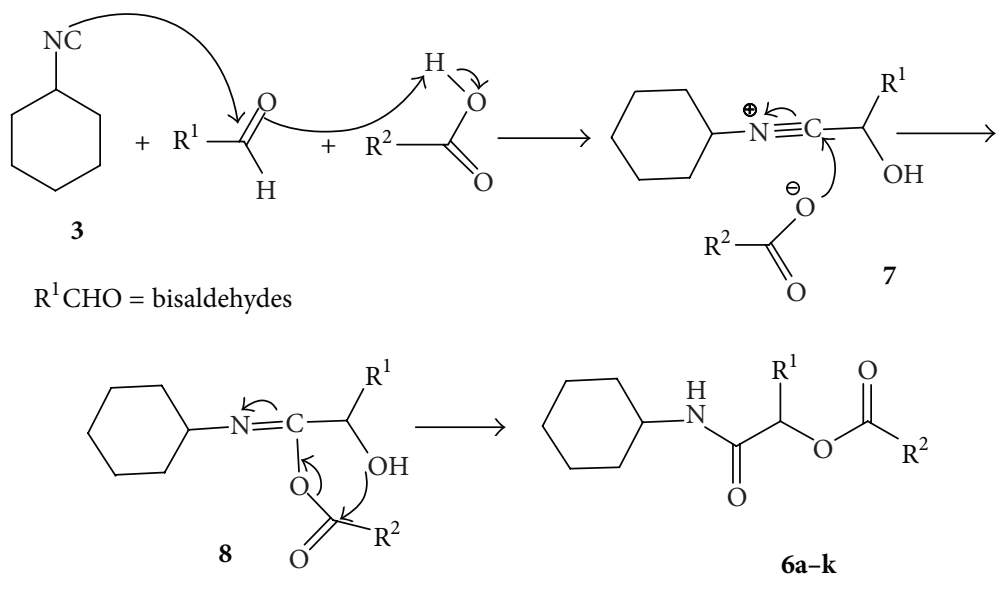

Scheme 2: Proposed mechanism for the formation of 1,1'-(aryl)bis(2-(cyclohexylamino)-2-oxoethane-1,1-diyl) di(alkanoate and benzoate) derivatives.

and Bacillus subtilis ATCC 6633 as a gram-positive model. Compounds $\mathbf{6 a}-\mathbf{c}$ and $\mathbf{6 h}-\mathbf{j}$ exhibited good antibacterial activity against Pseudomonas aeruginosa that may be due to the incorporation of amide and carbonyl groups. Also the results indicate that compounds $\mathbf{6 a}, \mathbf{6 b}, \mathbf{6 d}, \mathbf{6 j}$, and $\mathbf{6 k}$ have moderate and $\mathbf{6} f$ hasa good growth-inhibiting activity against Bacillus subtilis. Among these substances, $\mathbf{6 a}-\mathbf{c}, \mathbf{6 g}$, and $\mathbf{6 k}$ show low growth-inhibiting effects on Acinetobacter calcoaceticus. The results are shown in Table 2.

\section{Conclusions}

In summary, we have developed an expeditious and efficient protocol for the synthesis of novel $1,1^{\prime}$-(aryl)bis(2(cyclohexylamino)-2-oxoethane-1,1-diyl) di(alkanoate and benzoate) derivatives $(6 \mathbf{a}-\mathbf{k})$ via the reaction of alkyl and aryl carboxylic acids, various bisaldehydes, and isocyanocyclohexane in water under ultrasonic irradiation using onepot three-component approach. The reaction gives excellent yields $(87-96 \%)$ of the products in short reaction times (4$5 \mathrm{~min}$ ). The reaction is clean and highly expeditious, involves water as the green solvent, and occurs at ambient conditions. The protocol does not involve the use of catalyst and tedious workup procedure and thus is an environmentally friendly process. Some of the compounds prepared as part of this study exhibited good antibacterial activity against Pseudomonas aeruginosa.

\section{Conflict of Interests}

The authors declare that there is no conflict of interests.

\section{Acknowledgments}

The authors thank the Research Committee of University of Guilan for the partial support of this study.

\section{References}

[1] A. Baeyer and V. Drewsen, "Darstellung von indigblau aus orthonitrobenzaldehyd," Chemische Berichte, vol. 15, pp. 2856$2864,1882$.

[2] P. Ball, $\mathrm{H}_{2} \mathrm{O}: \mathrm{A}$ Biography of Water, Phoenix Press, London, UK, 2000.

[3] A. Chanda and V. V. Fokin, "Organic synthesis 'on water", Chemical Reviews, vol. 109, no. 2, pp. 725-748, 2009.

[4] T. Head-Gordon and G. Hura, "Water structurefrom scattering experiments and simulation," Chemical Reviews, vol. 102, no. 8 , pp. 2651-2670, 2002.

[5] M. C. Bellissent-Funnel and J. C. Done, Hydrogen Bond Networks, Kluwer Academic Publishers, Boston, Mass, USA, 1994.

[6] Y. Jung and R. A. Marcus, "On the theory of organic catalysis 'on water"' Journal of the American Chemical Society, vol. 129, no. 17, pp. 5492-5502, 2007.

[7] M. C. Pirrung and K. Das Sarma, "Multicomponent reactions are accelerated in water," Journal of the American Chemical Society, vol. 126, no. 2, pp. 444-445, 2004.

[8] S. Narayan, J. Muldoon, M. G. Finn, V. V. Fokin, H. C. Kolb, and K. B. Sharpless, "On water': unique reactivity of organic compounds in aqueous suspension," Angewandte ChemieInternational Edition, vol. 44, no. 21, pp. 3275-3279, 2005.

[9] Y. Hayashi, "In water or in the presence of water?" Angewandte Chemie-International Edition, vol. 45, no. 48, pp. 8103-8104, 2006.

[10] A. Dömling, "Recent advances in isocyanide-based multicomponent chemistry," Current Opinion in Chemical Biology, vol. 6, no. 3, pp. 306-313, 2002.

[11] T. Bousquet, M. Jida, M. Soueidan, R. Deprez-Poulain, F. Agbossou-Niedercorn, and L. Pelinski, "Fast and efficient solvent-free Passerini reaction," Tetrahedron Letters, vol. 53, no. 3, pp. 306-308, 2012.

[12] J. E. Biggs-Houck, A. Younai, and J. T. Shaw, "Recent advances in multicomponent reactions for diversity-oriented synthesis," Current Opinion in Chemical Biology, vol. 14, no. 3, pp. 371-382, 2010. 
[13] L. Weber, "The application of multi-component reactions in drug discovery," Current Medicinal Chemistry, vol. 9, no. 23, pp. 2085-2093, 2002.

[14] A. Dömling and I. Ugi, "Multicomponent reactions with isocyanides," Angewandte Chemie -International Edition, vol. 39, no. 18, pp. 3169-3210, 2000.

[15] A. Dondoni and A. Massi, "Design and synthesis of new classes of heterocyclic C-glycoconjugates and carbon-linked sugar and heterocyclic amino acids by Asymmetric Multicomponent Reactions (AMCRs)," Accounts of Chemical Research, vol. 39, no. 7, pp. 451-463, 2006.

[16] L. A. Wessjohann, D. G. Rivera, and O. E. Vercillo, "Multiple multicomponent macrocyclizations (MiBs): a strategie development toward macrocycle diversity," Chemical Reviews, vol. 109, no. 2, pp. 796-814, 2009.

[17] M. M. Heravi and S. Moghimi, "Catalytic multicomponent reactions based on isocyanides," Journal of the Iranian Chemical Society, vol. 8, no. 2, pp. 306-373, 2011.

[18] J. E. Biggs-Houck, A. Younai, and J. T. Shaw, "Recent advances in multicomponent reactions for diversity-oriented synthesis," Current Opinion in Chemical Biology, vol. 14, no. 3, pp. 371-382, 2010.

[19] A. Golisade, J. Wiesner, C. Herforth, H. Jomaa, and A. Link, "Anti-malarial activity of N6-substituted adenosine derivatives. Part I," Bioorganic \& Medicinal Chemistry, vol. 10, no. 3, pp. 769777, 2002.

[20] P. T. Anastas and J. C. Warner, Green Chemistry, Theory and Practice, Oxford University Press, New York, NY, USA, 1998.

[21] T. Sela and A. Vigalok, "Salt-controlled selectivity in "on water" and "in water" Passerini-type multicomponent reactions," Advanced Synthesis \& Catalysis, vol. 354, pp. 2407-2411, 2012.

[22] C. Cui, C. Zhu, X. J. Du, Z. P. Wang, Z. M. Li, and W. G. Zhao, "Ultrasound-promoted sterically congested Passerini reactions under solvent-free conditions," Green Chemistry, vol. 14, pp. 3157-3163, 2012.

[23] T. J. Mason, "Ultrasound in synthetic organic chemistry," Chemical Society Reviews, vol. 26, no. 6, pp. 443-451, 1997.

[24] L. Pizzuti, P. L. G. Martins, B. A. Ribeiro et al., "Efficient sonochemical synthesis of novel 3,5-diaryl-4,5-dihydro- $1 \mathrm{H}$ pyrazole-1-carboximidamides," Ultrasonics Sonochemistry, vol. 17, no. 1, pp. 34-37, 2010.

[25] M. Nikpassand, M. Mamaghani, F. Shirini, and K. Tabatabaeian, "A convenient ultrasound-promoted regioselective synthesis of fused polycyclic 4-aryl-3-methyl-4,7-dihydro-1H-pyrazolo[3,4b]pyridines," Ultrasonics Sonochemistry, vol. 17, no. 2, pp. 301$305,2010$.

[26] B. Karmakar and J. Banerji, "An expedient, fast and competent synthesis of organic dithiocarbamates over nanocrystalline $\mathrm{MgO}$ in water at room temperature," Tetrahedron Letters, vol. 52, no. 49, pp. 6584-6586, 2011.

[27] C. J. Li, “Aqueous Barbier-Grignard type reaction," Chemical Reviews, vol. 93, pp. 2023-2035, 1993.

[28] U. M. Lindström, "Stereoselective organic reactions in water," Chemical Reviews, vol. 102, no. 8, pp. 2751-2772, 2002.

[29] S. Kobayashi and K. Manabe, "Development of novel Lewis acid catalysts for selective organic reactions in aqueous media," Accounts of Chemical Research, vol. 35, no. 4, pp. 209-217, 2002.

[30] C.-J. Li, "Organic reactions in Aqueous media with a focus on carbon-carbon bond formations: a decade update," Chemical Reviews, vol. 105, no. 8, pp. 3095-3165, 2005.
[31] C.-J. Li and L. Chen, "Organic chemistry in water," Chemical Society Reviews, vol. 35, no. 1, pp. 68-82, 2006.

[32] U. M. Lindstrom, Organic Reactions in Water: Principles, Strategies and Applications, Blackwell, Oxford, UK, 2007.

[33] M. Mamaghani, A. Loghmanifar, and M. R. Taati, "An efficient one-pot synthesis of new 2-imino-1,3-thiazolidin-4-ones under ultrasonic conditions," Ultrasonics Sonochemistry, vol. 18, no. 1, pp. 45-48, 2011.

[34] A. Azimi Roshan, M. Mamaghani, N. O. Mahmoodi, and F. Shirini, "An efficient regioselective sonochemical synthesis of novel 4-aryl-3-methyl-4,5-dihydro-1H-pyrazolo[3,4b]pyridin-6(7H)-ones," Chinese Chemical Letters, vol. 23, no. 4, pp. 399-402, 2012.

[35] R. Hossein nia, M. Mamaghani, K. Tabatabaeian, F. Shirini, and M. Rassa, "An expeditious regioselective synthesis of novel bioactive indole-substituted chromene derivatives via one-pot three-component reaction," Bioorganic \& Medicinal Chemistry, vol. 22, pp. 5956-5960, 2012.

[36] M. Mamaghani and S. Dastmard, "An efficient ultrasoundpromoted synthesis of the Baylis-Hillman adducts catalyzed by imidazole and l-proline," Ultrasonics Sonochemistry, vol. 16, no. 4, pp. 445-447, 2009. 

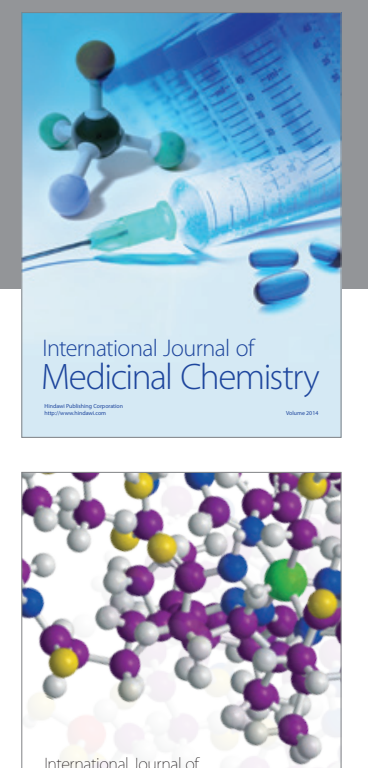

\section{Carbohydrate} Chemistry

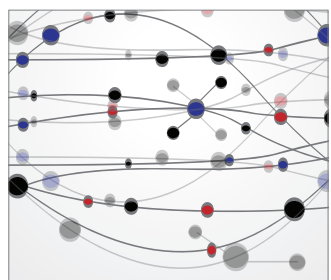

The Scientific World Journal
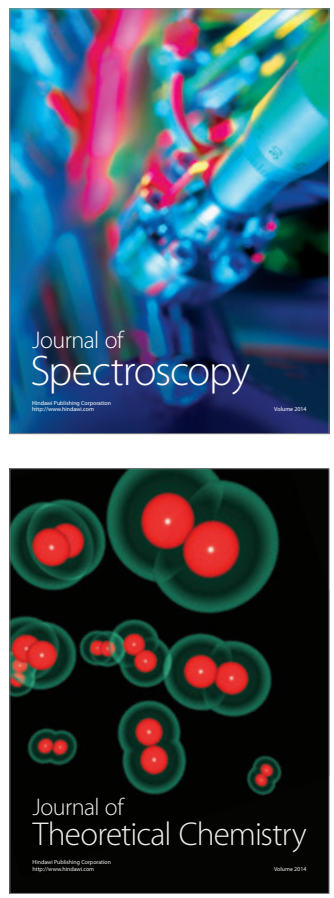
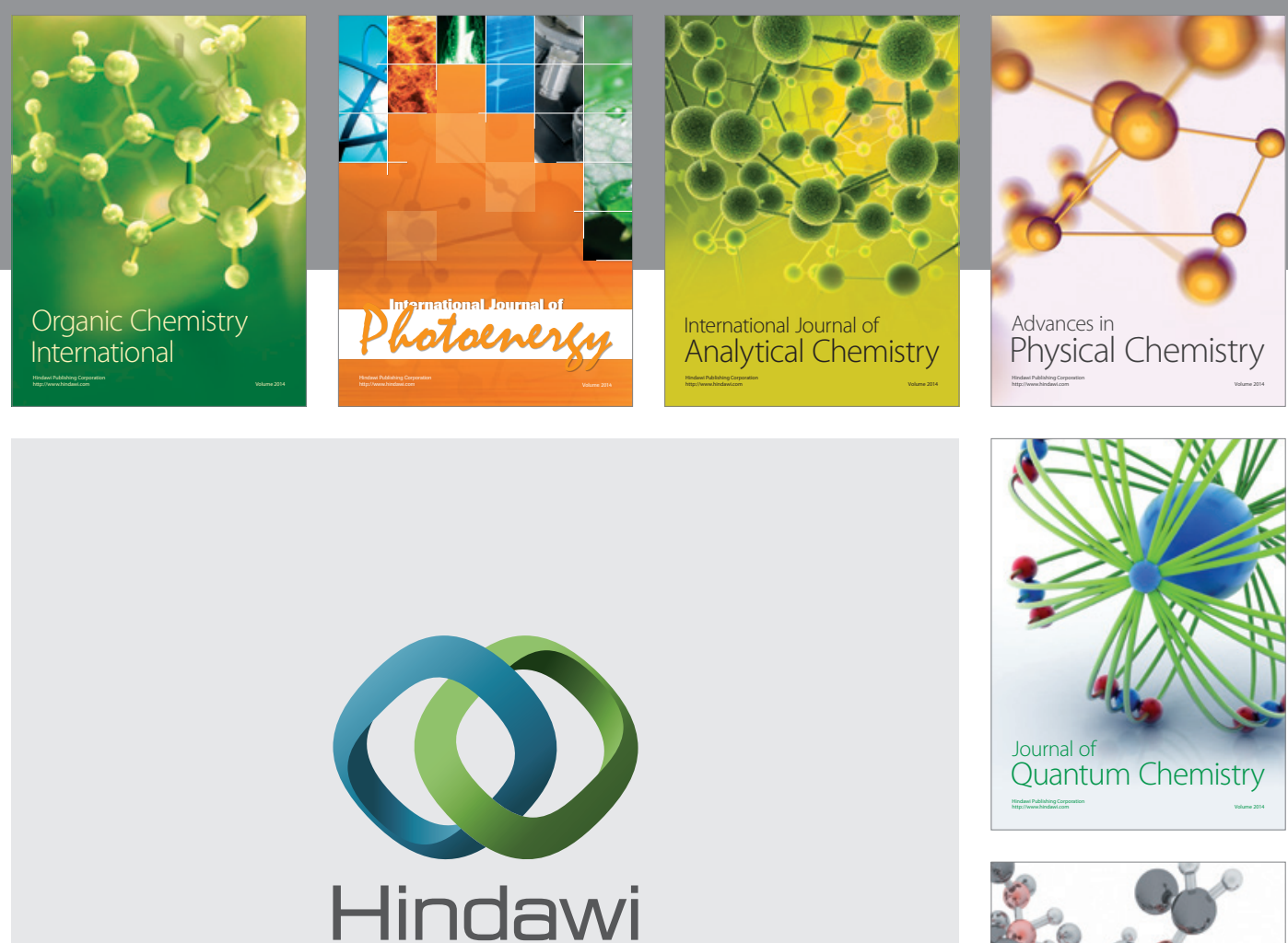

Submit your manuscripts at

http://www.hindawi.com

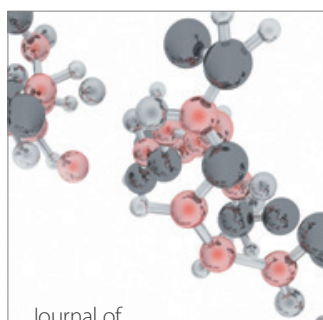

Analytical Methods

in Chemistry

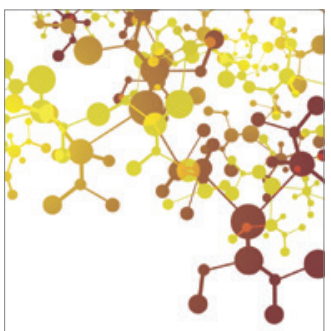

Journal of

Applied Chemistry

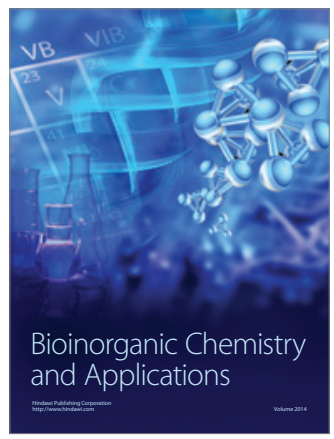

Inorganic Chemistry
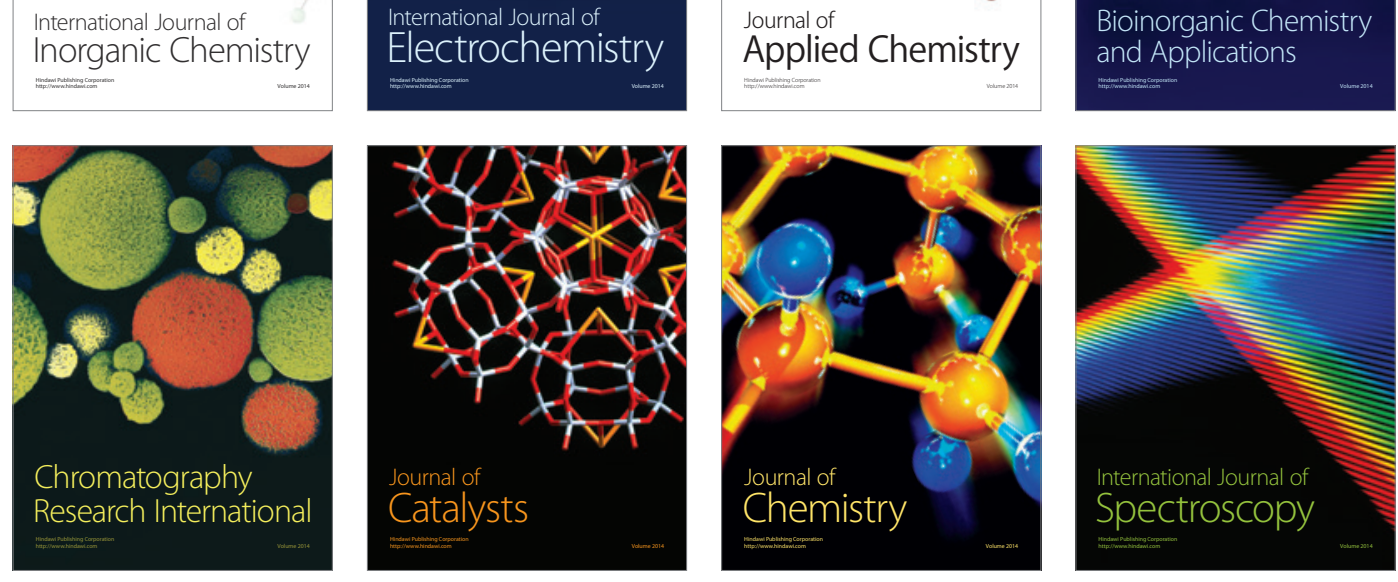